\title{
Acute telogen effluvium associated with SARS-CoV-2 infection
}

Miguel Domínguez-Santás, Lara HayaMartínez, Diego Fernández-Nieto, Juan Jiménez-Cauhé, Ana Suárez-Valle, Borja Díaz-Guimaraens

SARS-COV-2 is known to produce diverse acute cutaneous manifestations. ${ }^{1}$ This article reports a dermatological finding as a sequela of COVID-19.

A woman aged 42 years presented to our dermatology department in July 2020. She reported a dramatic and abrupt drop in hair volume that had started two weeks prior and was causing her great emotional distress. She was specifically concerned about the large number of hairs she was shedding every time she washed her hair. She also reported that she fell ill and tested positive for SARS-CoV-2 in April 2020. A real-time reverse transcription polymerase chain reaction (rRT-PCR) for SARS-CoV-2 was performed from a nasopharyngeal swab, rendering a positive result. In terms of COVID-19 symptoms, she had experienced coughing and a fever of up to $39^{\circ} \mathrm{C}$ that lasted for two weeks. She did not require hospitalisation or any specific treatment.

Dermatological examination showed a diffuse loss of hair volume without any defined alopecic patches (Figure 1). She did not present with scaling, erythema or any other dermatological abnormalities on her scalp. A pull test was strongly positive (performed by wrapping 60 hairs between the thumb and index fingers and pulling gently upwards; it is considered positive when more than two hairs are removed). Trichoscopy (dermoscopy of scalp hairs) was unspecific, with numerous follicular units of only one hair and without anisotrichosis (hair shafts with different diameters, typical of androgenic alopecia). There was no loss of follicular units (a sign of cicatricial alopecia). There were no trichoscopic signs of alopecia areata or other kinds of alopecia (Figure 2). A trichogram (the plucking of 40-60 hairs and posterior microscopic analysis of the hair bulbs to discern how many of them are in anagen or telogen phase, considered consistent with telogen effluvium if more than $25 \%$ are in telogen phase) was performed, showing $35 \%$ of telogen follicles. ${ }^{2}$

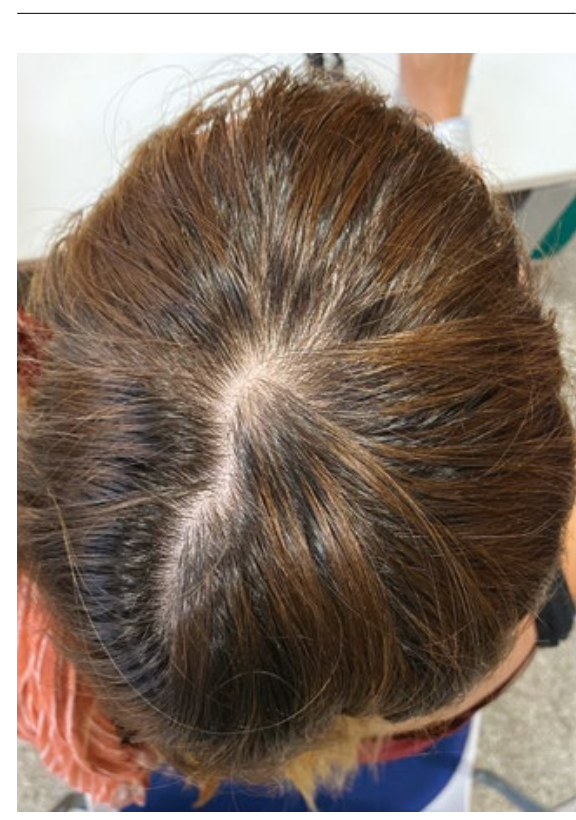

Figure 1. Diffuse loss of hair volume without any defined alopecic patches
The patient was diagnosed as having acute telogen effluvium. We hypothesise that the trigger could have been her SARS-CoV-2 infection three months prior to this event. No treatment for telogen effluvium was given to the patient. A careful explanation - reassuring her that since the trigger had already been resolved, her hair would resume normal growth - was enough to calm her unease.

Acute telogen effluvium is a common entity, caused by the abrupt and synchronic transit of at least $20 \%$ of hairs from anagen phase (the active growth phase of hair follicles) to telogen phase

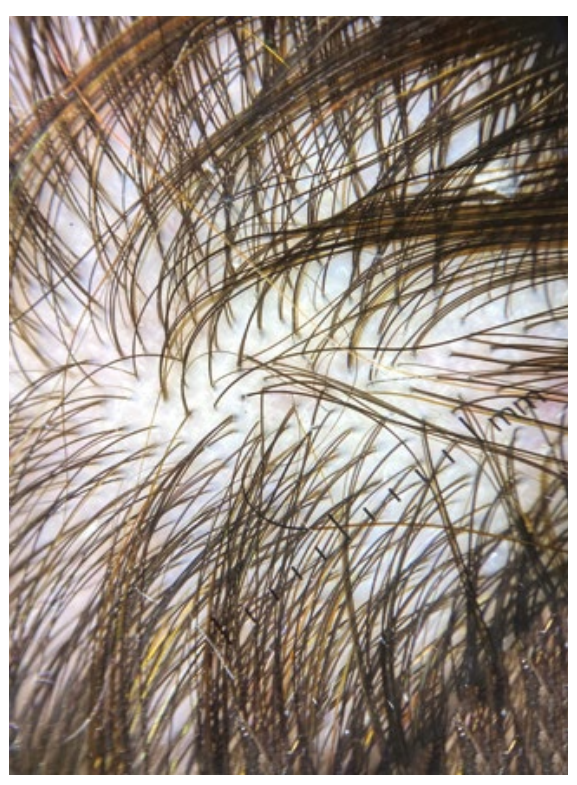

Figure 2. Numerous follicular units of only one hair and without anisotrichosis (hair shafts with different diameters); no trichoscopic signs of alopecia areata or other kinds of alopecia 
(the resting phase of hair follicles, after which the hair shafts are shed), leading to physiological hair loss approximately 3-4 months after a stressing triggering cause. Other aetiologies that may lead to acute telogen effluvium are surgeries, diets and other stressing pathologies or infections. ${ }^{2}$ Patients typically report significant hair loss and a decrease in hair volume (they commonly complain about their ponytail reducing in diameter) without well-defined alopecic patches. A pull test is typically positive. ${ }^{2}$ The main differential diagnosis in this case (a diffuse non-scarring alopecia) would be androgenic alopecia (trichoscopy would typically show anisotrichosis, with more than $10 \%$ of miniaturised hairs in the frontal area; this was not seen in our patient), diffuse alopecia areata (trichoscopy would typically show signs of alopecia areata, such as black or yellow dots, exclamation mark hairs or tapered hairs) or chronic telogen effluvium (defined as a telogen effluvium that lasts for $>6$ months, sometimes due to iron deficiency or hypothyroidism, among other causes). ${ }^{2-4}$

The clinical presentation and the evidence of a well-defined trigger is almost always diagnostic of telogen effluvium and does not require biopsy (as in this case). When there are doubts about other possible aetiologies, a punch biopsy from the scalp can be taken. Dermatologists usually perform two punch biopsies for the diagnosis of challenging alopecias, one for vertical sections and another one for horizontal sections. The histopathology of telogen effluvium would typically show a normal total number of hairs with an increase in the telogen count to $>20 \%$ of hairs and an absence of scarring or inflammation. ${ }^{2}$

There is no specific treatment for acute telogen effluvium, as it is a reversible type of alopecia and resolves after cessation of the trigger. ${ }^{2}$

By sharing this case, we hope to improve the knowledge of non-dermatologist physicians on acute telogen effluvium, a cause of common dermatological consultation that will likely become even more frequent because of the stressing events associated with the pandemic (both the infection and the psychological stress of confinement). Reassuring patients that it is a transitory condition is key.

First published online 26 August 2020.

\section{Authors}

Miguel Domínguez-Santás MD, Dermotologist, Dermatology Department, Hospital Universitario Ramón y Cajal, Madrid, Spain. miguelds92@hotmail.com

Lara Haya-Martínez MD, Dermotologist, Dermatology Department, Fundación Jiménez Díaz, Madrid, Spain

Diego Fernández-Nieto MD, Dermotologist,

Dermatology Department, Hospital Universitario

Ramón y Cajal, Madrid, Spain

Juan Jiménez-Cauhé MD, Dermotologist,

Dermatology Department, Hospital Universitario Ramón y Cajal, Madrid, Spain

Ana Suárez-Valle MD, Dermotologist, Dermatology Department, Hospital Universitario Ramón y Cajal, Madrid, Spain

Borja Díaz-Guimaraens MD, Dermotologist, Dermatology Department, Hospital Universitario Ramón y Cajal, Madrid, Spain

Competing interests: None.

Provenance and peer review: Not commissioned, externally peer reviewed.

Citation: Domínguez-Santás M, Haya-Martínez L, Fernández-Nieto $D$, Jiménez-Cauhé J, Suárez-Valle A, Díaz-Guimaraens B. Acute telogen effluvium associated with SARS-CoV-2 infection. Aust $J$ Gen Pract 2020;49 Suppl 32. doi: 10.31128/AJGPCOVID-32.

\section{References}

1. Galván Casas C, Català A, Carretero Hernández $G$, et al. Classification of the cutaneous manifestations of COVID-19: A rapid prospective nationwide consensus study in Spain with 375 cases. Br J Dermatol 2020;183(1):71-77. doi: 10.1111/bjd.19163.

2. Asghar F, Shamim N, Farooque U, Sheikh H, Aqeel R. Telogen effluvium: A review of the literature. Cureus 2020;12(5):e8320. doi: 10.7759/ cureus.8320.

3. Alessandrini A, Starace M, Bruni F, et al. Alopecia areata incognita and diffuse alopecia areata: Clinical, trichoscopic, histopathological, and therapeutic features of a 5-year study. Dermatol Pract Concept 2019;9(4):272-77. doi: 10.5826/ dpc.0904a05.

4. Starace M, Orlando G, Alessandrini A Piraccini BM. Female androgenetic alopecia: An update on diagnosis and management. Am J Clin Dermatol 2020;21(1):69-84. doi: 10.1007/s40257019-00479-x. 